\title{
The Diophantine Approximation of Roots of Positive Integers*
}

\section{Charles F. Osgood**}

(September 21, 1970)

The following result is established:

THEOREM: Suppose that $\mathrm{k} \geqslant 150$ and $\mathrm{m}$ are fixed positive integers. Then

$$
\left|\sqrt[k]{m}-\mathrm{pq}^{-1}\right|<\mathrm{q}^{-\frac{7}{8} k}
$$

can hold for at most one pair of relatively prime positive integers $\mathrm{p}$ and $\mathrm{q}$ with $\mathrm{q} \geqslant 2^{9}(\sqrt[k]{m}+1)^{6}$.

The new feature of this result is that the lower bound on $q$ is given explicitly and is "small."

Keywords: Diophantine approximation; diophantine equation; effective computability.

\section{Introduction}

Recently Schinzel [4] ${ }^{1}$ and Davenport [1] have each obtained a result of the following sort: Let $\alpha$ be a real algebraic number of degree $r \geqslant 2$. Let $s$ be a positive real number larger than $s(r)$, where for Davenport $r>s(r)=\frac{1}{2} r+0(1)>\frac{1}{2} r$ while for Schinzel $s(r)=3(r / 2)^{1 / 2}$. Then there exists an effectively computable positive integer $q_{0}(\alpha, s)$ such that, with at most one exception, every pair of relatively prime integers $p$ and $q$ with $q \geqslant q_{0}(\alpha)$ satisfies the inequality

$$
\left|\alpha-p q^{-1}\right| \geqslant \frac{1}{2} q^{-s}
$$

(Also Seppo Hyyrö in [2] obtained something analogous to Davenport's result for kth roots of rational numbers, where $k \geqslant 2$ is a positive integer.)

None of these authors, however, calculated $q_{0}(\alpha, s)$ explicitly for any class of $\alpha$ and $s$. With the aid of a theorem in a recent paper by the present author we can obtain explicitly a rather "small" $q_{0}(\alpha, s)$ for a certain class of $\alpha$ and $s$. (Below one could drop the lower bound on $k$ very considerably by allowing a larger $s<k$ and a larger $q_{0}(\alpha, s)$.)

Theorem I: Suppose that $\mathrm{k} \geqslant 150$ and $\mathrm{m}$ are fixed positive integers. Then

$$
\left|\sqrt[k]{m}-\mathrm{pq}^{-1}\right|<\mathrm{q}^{-\frac{7}{8} \mathrm{k}}
$$

can hold for at most one pair of relatively prime positive integers $\mathrm{p}$ and $\mathrm{q}$ with $\mathrm{q} \geqslant 2^{9}(\sqrt[k]{\mathrm{m}}+1)^{6}$.

DEFINITIONS: By a reduced approximation we shall mean a fraction $\frac{\mathrm{p}}{\mathrm{q}}$ where $\mathrm{p}$ and $\mathrm{q}$ are positive integers and $(\mathrm{p}, \mathrm{q})=1$. Set $\theta(\beta)=\frac{\mathrm{k}}{6}\left(\left(\frac{7}{8} \mathrm{k}-2\right)^{\beta}-15\right)$.

AMS subject classification. Primary 10F05.

*An invited paper. This paper was written in part while the author was on a postdoctual Reseäch Associateship at the National Bureau of Standards, Wash. ington, D.C. (This award is given by the National Bureau of Standards in association with the National Academy of Sciences and the National Research Council.)

**Present address: Naval Research Laboratory, Washington, D.C. 20390.

${ }^{1}$ Figures in brackets indicate the literature references at the end of this paper. 
THEOREM II: If $\mathrm{m}$ and $\mathrm{k} \geqslant 150$ are positive integers and $\beta$ is any positive integer such that $\mathrm{m}<2^{\theta(\beta)}$ then (1) is satisfied by at most $\beta+1$ distinct reduced approximations.

THEOREM III: If $\mathrm{k} \geqslant 150$ and $\mathrm{m}<\left(\mathrm{q}_{0}{ }^{\frac{7}{8}}{ }^{\mathrm{k}-1} 2^{-16}\right)^{\frac{k}{6}}$ are positive integers then (1) is satisfied by at most two distinct reduced fractions with denominators larger than or equal to $\mathrm{q}_{0} \geqslant 2$.

Setting $q_{0} \geqslant 10$ in Theorem III we obtain,

Corollary: If $\mathrm{k} \geqslant 150$ and $\mathrm{m}$ are positive integers with $\sqrt[\mathrm{k}]{\mathrm{m}}<10^{20}$ then (1) is satisfied by at most two distinct reduced fractions with denominators larger than 9.

\section{Section I}

Proof of Theorem I. We shall use the Theorem of [3] (which will be stated below for the case $\left.\mathrm{k}_{1}=1, \mathrm{n}=2, \epsilon=2\right)$ and the supposed existence of two solutions of $(1), \mathrm{p}^{\prime}, \mathrm{q}^{\prime}$ and $\mathrm{p}^{\prime \prime}, \mathrm{q}^{\prime \prime}$ where $\left(\mathrm{p}^{\prime}, \mathrm{q}^{\prime}\right)=\left(\mathrm{p}^{\prime \prime}, \mathrm{q}^{\prime \prime}\right)=1$ and $\mathrm{q}^{\prime \prime}>\mathrm{q}^{\prime} \geqslant 2^{9}(\sqrt[k]{\mathrm{m}}+1)^{6}$. Let

From [3] we have: Let $s$ and $k$ be positive integers, and $k \geqslant 2$. Let $0<\epsilon<+\infty$ be a real number.

$$
K=2^{\frac{3}{2} k+\frac{1}{2}} s^{2}
$$

Let $N$ denote a positive integer larger than $K$. Set

and

$$
1>\Lambda(N)=\frac{\log \left(N K^{-1}\right)}{\log \left(2^{7} N K\right)}>0,
$$

$$
\varphi(N)=\left(2^{7} K N\right)^{6+3 k-1} .
$$

Let $q$ denote a positive integer and $\left(p_{1}, p_{2}\right)$ a nonzero vector of nonnegative integers. Let $C$ denote any real number satisfying $0 \leqslant C \leqslant 1$. Then we have,

THEOREM: If $q>\varphi(N)$

$$
\max \left\{\left|C N^{k^{-1}}-p_{1} q^{-1}\right|,\left|C(N+s)^{k^{-1}}-p_{2} q^{-1}\right|\right\} \geqslant \frac{1}{2}(2 q)^{-\left(1+\frac{3}{\Lambda(N)}\right)}
$$

for all $C$ and $\left(p_{1}, p_{2}\right)$.

Returning to the proof of Theorem I, if $\left|\sqrt[k]{m}-p^{\prime}\left(q^{\prime}\right)^{-1}\right|<\left(q^{\prime}\right)^{-\frac{7}{8} k}$ then $\left|\left(q^{\prime}\right)^{k} m-\left(p^{\prime}\right)^{k}\right|$ $<k(\sqrt[k]{m}+1)^{k-1}\left(q^{\prime}\right)^{\frac{k}{8}}$. Choose $N$ to be the smaller of $m\left(q^{\prime}\right)^{k}$ and $\left(p^{\prime}\right)^{k}$. (Then $m\left(q^{\prime}\right)^{k} \geqslant N>m\left(q^{\prime}\right)^{k}$ $-k(\sqrt[k]{m}+1)^{k+1}\left(q^{\prime}\right)^{\frac{k}{8}}$. Set $s=\left|\left(q^{\prime}\right)^{k} m-\left(p^{\prime}\right)^{k}\right|$.

Note that then

$$
K<2^{\frac{3}{2} k+\frac{1}{2}} k^{2}(\sqrt[k]{m}+1)^{2 k-2}\left(q^{\prime}\right)^{\frac{k}{4}}
$$

We shall presently show that $N>K$. Assuming $N>K$ for the present and setting $C=1$, we see that by the Theorem from [3],

$$
\left|\sqrt[k]{m}-p q^{-1}\right| \geqslant\left(2 q^{\prime}\right)^{-1}(2 q)^{-\left(1+\frac{3}{\Lambda(N)}\right)}
$$

for all positive integers $p$ and $q$ with $q>\left(2^{7} K N\right)^{6+3 k^{-1}}$. We shall eventually contradict (3) with $p=r p^{\prime \prime}, q=r q^{\prime \prime}$ for a positive integer $r \geqslant 2$.

First to obtain lower bounds on $N$. Using $q^{\prime}>2^{9}(\sqrt[k]{m}+1)^{6}$ and $k \geqslant 150$ we see that

Also, trivially,

$$
m\left(q^{\prime}\right)^{k}>2\left(2^{3 k+1} k^{4}(\sqrt[k]{m}+1)^{4 k-4}\right)\left(q^{\prime}\right)^{k / 2} .
$$

$$
k(\sqrt[k]{m}+1)^{k-1}\left(q^{\prime}\right)^{k / 8}<2^{3 k+1} k^{4}(\sqrt[k]{m}+1)^{4 k-4}\left(q^{\prime}\right)^{k / 2} .
$$


Thus

$$
N>m\left(q^{\prime}\right)^{k}-k(\sqrt[k]{m}+1)^{k-1}\left(q^{\prime}\right)^{k / 8}>2^{3 k+1} k^{4}(\sqrt[k]{m}+1)^{4 k-4}\left(q^{\prime}\right)^{k / 2}>K^{2}>K .
$$

The last two inequalities come from (2).

Using the triangle inequality we conclude that

$$
2\left(q^{\prime}\right)^{-\frac{7}{8} k}>\left|\frac{p^{\prime}}{q^{\prime}}-\frac{p^{\prime \prime}}{q^{\prime \prime}}\right|>\left(q^{\prime} q^{\prime \prime}\right)^{-1} ; \quad \text { thus, } \quad q^{\prime \prime}>\frac{1}{2}\left(q^{\prime}\right)^{\frac{7}{8} k-1} \stackrel{\text { def. }}{=} M_{1}
$$

Now

$$
1+\frac{3}{\Lambda(N)}=4+\frac{3 \log \left(2^{7} K^{2}\right)}{\log \left(N K^{-1}\right)}<4+\frac{3 \log \left(2^{7} K^{2}\right)}{\log K}
$$

because $N>K^{2}$ as we saw in (4). Thus

$$
1+\frac{3}{\Lambda(N)}<10+\frac{3 \log 2^{7}}{\log K}<10 \frac{1}{10}
$$

since $K=2^{\frac{3}{2} k+\frac{1}{2}} s^{2}>2^{225}$. Now since $q^{\prime}<q^{\prime \prime}$ and $k \geqslant 150$,

$$
\left(2 q^{\prime}\right)^{-1}\left(2 q^{\prime \prime}\right)^{-10.1}>\left(q^{\prime \prime}\right)^{-\frac{7}{8} k}
$$

so by the Theorem from [3], by formula (2), and by our bound on $N$,

$$
q^{\prime \prime}<\left(2^{7} K N\right)^{6+3 k^{-1}} \leqslant\left(m(\sqrt[k]{m}+1)^{2 k-2} 2^{7^{1 / 2}+\frac{3}{2} k} k^{2}\left(q^{\prime}\right)^{\frac{5}{4} k}\right)^{6+3 k^{-1}} \stackrel{\text { def. }}{=} M_{2} .
$$

Let ${ }^{2} r=\left[M_{2} M_{1}^{-1}\right]+1$ and $\delta=\frac{\log \left(q^{\prime \prime}\right)}{\log \left(2 r q^{\prime \prime}\right)}$. Now $\left|\sqrt[k]{m}-\frac{r p^{\prime \prime}}{r q^{\prime \prime}}\right|<\left(2 r q^{\prime \prime}\right)^{-\frac{7}{8} \delta k}$.

Further $\delta \geqslant\left[\log \left(q^{\prime \prime}\right)\right]\left[\log \left(4 M_{2} M_{1}^{-1} q^{\prime \prime}\right)\right]^{-1}>\left[\log \left(M_{1}\right)\right]\left[\log \left(4 M_{2}\right)\right]^{-1}$, since $q^{\prime \prime}>M_{1}$.

We wish to show that

$$
\left.\left[\frac{7}{8} k \log \left(\frac{1}{2}\left(q^{\prime}\right)^{\frac{7}{8} k-1}\right)\right]\left[\log \left(4 m(\sqrt[k]{m}+1)^{2 k-2} 2^{71 / 2+\frac{3}{2} k} k^{2}\left(q^{\prime}\right)^{\frac{5}{4} k}\right)^{6+3 k^{-1}}\right)\right]^{-1} \geqslant 10 \frac{1}{5} .
$$

This would give us our desired contradiction since by (3) and (5) and the inequalities $q^{\prime \prime}>\frac{1}{2}\left(q^{\prime}\right)^{\frac{7}{8} k-1}$ and $k \geqslant 150$

$$
\left|\sqrt[k]{m}-\frac{r p^{\prime \prime}}{r q^{\prime \prime}}\right|>\left(2 q^{\prime}\right)^{-1}\left(2 r q^{\prime \prime}\right)^{-10.1}>\left(2 r q^{\prime \prime}\right)^{-10.2}
$$

while we would have that

$$
\left|\sqrt[k]{m}-\frac{r p^{\prime \prime}}{r q^{\prime \prime}}\right|<\left(2 r q^{\prime \prime}\right)^{-10.2}
$$

Since $m(\sqrt[k]{m}+1)^{2 k-2}<\left(q^{\prime}\right)^{\frac{k}{2}} 2^{-4 \frac{1}{2} k}$ and $k^{2}<2^{k}$ we see that $(6)$ is implied by

$$
\left.\frac{7}{8} k\left[\log \left(\frac{1}{2}\left(q^{\prime}\right)^{\frac{7}{8} k-1}\right)\right]\left[\log 4\left(\left(q^{\prime}\right)^{\frac{7}{4} k} 2^{-2 k+7^{\frac{1}{2}}}\right)^{6+3 k-1}\right)\right]^{-1} \geqslant 10 \frac{1}{5},
$$

${ }^{2}$ Here only we use the greatest integer notation. 
which is implied by

$$
\left[\frac{7}{8} k \log \left(\left(\frac{1}{2} q^{\prime}\right)^{\frac{7}{8} k-1}\right)\right]\left[\log \left(\left(\frac{1}{2} q^{\prime}\right)^{\frac{7}{4} k(6+3 k-1)}\right]^{-1} \geqslant 10 \frac{1}{5}\right.
$$

But then all that we have to see is that

$$
\frac{1}{2}\left(\frac{7}{8} k-1\right)\left(6+3 k^{-1}\right)^{-1} \geqslant 10 \frac{1}{5}
$$

if $k \geqslant 150$. This is easily done and proves Theorem I.

\section{Section II}

Proof of Theorem II: If $\frac{\mathrm{p}^{\prime}}{q^{\prime}}$ and $\frac{\mathrm{p}^{\prime \prime}}{q^{\prime \prime}}$ satisfy $(1)$ and $\mathrm{q}^{\prime \prime}>\mathrm{q}^{\prime}$ then either $\frac{\mathrm{p}^{\prime}}{q^{\prime}}=\frac{\mathrm{p}^{\prime \prime}}{q^{\prime \prime}}$ or $\mathrm{q}^{\prime \prime}>\frac{1}{2}\left(\mathrm{q}^{\prime}\right)^{\frac{7}{8} k-1}$. Thus if there is a $\beta+1-$ st reduced approximation (ordering them by the magnitude of their denominators) it has a denominator at least as large as $2^{\left(\frac{7}{8} k-2\right)^{\beta}} \geqslant 2^{15}(\sqrt[k]{m})^{6} \geqslant 2^{9}(\sqrt[k]{m}+1)^{6}$, so it is the final reduced approximation which satisfies ( 1 ).

Proof of Theorem III: If there are two reduced fractions $\frac{p^{\prime}}{q^{\prime}}$ and $\frac{p^{\prime \prime}}{q^{\prime \prime}}$ with $q^{\prime \prime}>q^{\prime} \geqslant q_{0}$ which satisfy (1) then

$$
q^{\prime \prime}>\frac{1}{2} q_{0}{ }^{\frac{7}{8} k-1}>2^{15}(\sqrt[k]{m})^{6} \geqslant 2^{9}(\sqrt[k]{m}+1)^{6},
$$

so these are all of the reduced approximations which satisfy (1).

\section{References}

[1] Davenport, H., A Note on Thue's Theorem, Mathematika, vol. 15, 76-87 (1968).

[2] Hyyrö, Seppo, Über Die Catalansche Problem, Ann. Acad. Scient. Fennicae, Series A, No. 355, 1-50 (1964).

[3] Osgood, C. F., The Simultaneous Approximation of Certain k-th Roots, Proc. Camb. Phil. Soc. 67, 75-86 (1970).

[4] Schinzel, Zentralblatt für Math., 137, 258 (1967).

(Paper 74B4-334) 